\title{
Phosphaturic mesenchymal tumor of the brain without tumor-induced osteomalacia in an 8-year-old girl: case report
}

\author{
Mark B. Ellis, DPM, ${ }^{1}$ Daniel Gridley, MD, ${ }^{2}$ Suresh Lal, MD, ${ }^{3}$ Geetha R. Nair, MD, ${ }^{4}$ and \\ Iman Feiz-Erfan, MD1
}

${ }^{1}$ Division of Neurosurgery and Departments of ${ }^{2}$ Radiology, ${ }^{3}$ Pediatrics, and ${ }^{4}$ Pathology, The University of Arizona College of Medicine-Phoenix, Maricopa Medical Center, Phoenix, Arizona

\begin{abstract}
Phosphaturic mesenchymal tumor (mixed connective tissue variant) (PMT-MCT) are tumors that may cause tumor-induced osteomalacia and rarely appear intracranially. The authors describe the case of an 8-year-old girl who was found to have PMT-MCT with involvement of the cerebellar hemisphere and a small tumor pedicle breaching the dura mater and involving the skull. This was removed surgically in gross-total fashion without further complication. Histologically the tumor was confirmed to be a PMT-MCT. There was no evidence of tumor-induced osteomalacia. At the 42-month followup, the patient is doing well, has no abnormalities, and is free of recurrence. PMT-MCTs are rare tumors that may involve the brain parenchyma. A gross-total resection may be effective to cure these lesions.
\end{abstract}

http://thejns.org/doi/abs/10.3171/2015.9.PEDS14617

KEY WORDS phosphaturic mesenchymal tumor; fibroblast growth factor 23; pediatric brain tumor; cerebellar tumor; oncology

$\mathrm{T}$ HE phosphaturic mesenchymal tumor (mixed connective tissue variant) (PMT-MCT) is a very rare, histopathologically distinct tumor that is frequently misdiagnosed as other mesenchymal tumors. ${ }^{14,31}$ PMTMCTs have been identified in a variety of soft tissues and osseous tissues, have a propensity to favor extremities and appendicular skeleton over the trunk or axial skeleton, 9,17,27 and are only reported to have intracranial involvement as an extension of the anterior skull base region or cavernous sinuses. ${ }^{13,4,6,8-10,17,22-25,28,30}$ PMT-MCTs are almost always associated with tumor-induced osteomalacia (TIO; also known as oncogenic osteomalacia); ;, ,22,25,30 however, PMTMCTs without known TIO have been reported to be histologically identical to typical PMT-MCTs. ${ }^{9}$ Expression of the FGF23 protein has been seen in $81 \%$ of immunohistologically analyzed samples and in $100 \%$ of those analyzed by reverse transcriptase polymerase chain reaction (RTPCR). ${ }^{9}$ The few reported cases of intracranial PMT-MCT in the literature ${ }^{3,6,8,23}$ were found close to paranasal sinuses in older patients (typically in the 3rd to 7th decades of life); only one of these tumors was without symptoms of TIO.

\section{Case Report}

History and Examination

An 8-year-old girl woke up with severe headaches. She was unable to walk due to severe imbalance. She was ataxic on physical examination. CT scanning showed a hemorrhagic and partially calcified brain tumor with obstructive hydrocephalus. The CT study also showed a small, bony calvarial defect in the posterior fossa associated with tumor (Fig. 1).

\section{Operation}

Resection via a right retrosigmoid approach with grosstotal resection was performed. Grossly, the tumor was a hemorrhagic, well-encapsulated, partially calcified lesion that could be resected in an en bloc fashion. At the time of surgery we found that the tumor had a pedicular attachment to the inner table of the skull. The outer table of the skull was grossly normal in appearance. Furthermore, the dura mater was defective in a circular fashion, approximately $7 \mathrm{~mm}$ in diameter. There was no frank invasion

ABBREVIATIONS ABC = aneurysmal bone cyst; PMT-MCT = phosphaturic mesenchymal tumor (mixed connective tissue variant); RT-PCR = reverse transcriptase polymerase chain reaction; $\mathrm{TIO}=$ tumor-induced osteomalacia.

SUBMITTED November 4, 2014. ACCEPTED September 14, 2015.

INCLUDE WHEN CITING Published online January 1, 2016; DOI: 10.3171/2015.9.PEDS14617. 

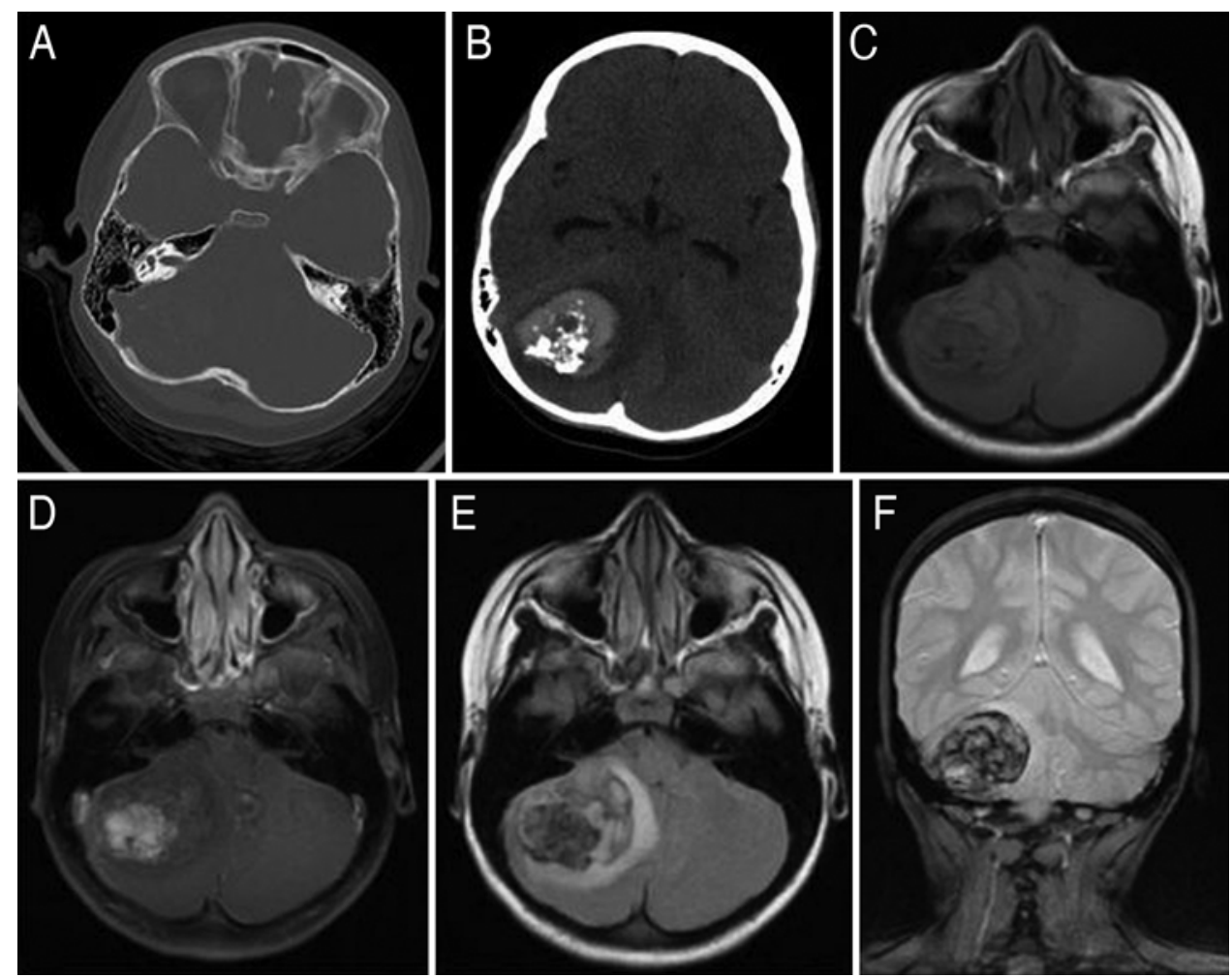

FIG. 1. Axial bone algorithm CT (A), axial soft-tissue algorithm CT (B), axial T1-weighted (C), axial T1-weighted postcontrast (D), axial FLAIR (E), and coronal T2*-weighted gradient echo (F) imaging of the posterior fossa reveals a hyperattenuating, heterogeneous T1 and T2 hypointense focus with internal enhancement involving the right cerebellar hemisphere with surrounding vasogenic edema. Axial CT imaging in bone algorithm demonstrates involvement of the right occipital calvarium.

of tumor into dura grossly. The area of bony and dura abnormality was removed. Postoperatively, the patient had an uncomplicated hospital course. The hydrocephalus resolved without shunting, and the patient was discharged home without neurological deficits.

\section{Histological Features}

Histopathological analysis was performed and identified a benign solid and cystic neoplasm with cytological and architectural features that were common in aneurysmal bone cysts (ABCs), hemangiopericytoma, and phosphaturic mesenchymal tumor. The tumor showed hyalinized stroma with prominent vascularity and cellular areas with oval- or spindle-shaped cells with oval or elongated nuclei showing no significant cytological atypia or mitotic activity. Scattered multinucleated osteoclast-like giant cells were present. There was mineralization of stroma with foci of "grungy" calcification and osteoid and chondroid features. The cells stained strongly positive for immunohistochemical stains vimentin and CD68 and were negative for epithelial membrane antigen, progesterone receptor, smooth muscle actin, and S100 (Fig. 2).

Molecular studies performed at Mayo Clinic showed that the tumor was positive for FGF23 mRNA expression by RT-PCR, consistent with the majority of PMTs. Fluorescence in situ hybridization (FISH) analysis of this tumor for USP6 fusion gene was negative. Approximately $70 \%$ of the ABC contained USP6 fusion genes.

\section{Postoperative Course}

Follow-up CT and MRI showed no residual tumor or abnormality. Repeat MRI at 2 years postresection showed no evidence of recurrent lesion. At the 42-month followup, the patient had resumed all normal activities, including basketball, with no deficits in vision, balance, strength, or coordination, and she is without headaches. Furthermore, there continues to be thus far no clinical concern for osteomalacia.

\section{Discussion}

PMT-MCT is a rare tumor of soft tissue and bone that is most often associated with a distinct paraneoplastic syndrome known as oncogenic osteomalacia or $\mathrm{TIO}^{21}$ with associated phosphaturia, hypophosphatemia, and osteomalacia. It is estimated that $53 \%$ occur in bone, $45 \%$ in soft tissue, and 3\% in skin. ${ }^{27}$ Weidner and Santa Cruz first coined the term "phosphaturic mesenchymal tumor" in $19877^{29}$ and developed a classification of 4 main subtypes: primitive-appearing mixed connective tissue tumors, osteoblastoma-like, nonossifying fibroma-like, and ossifying fibroma-like. In 2004, Folpe et al. ${ }^{9}$ reported on 32 cases and preformed a thorough review of all literature. They compared the clinical features and diagnoses, offering revised diagnoses when applicable, and found immunohistochemistry and RT-PCR identification of FGF23 to be a crucial part of the diagnosis ( $81 \%$ and $100 \%$, respectively, 

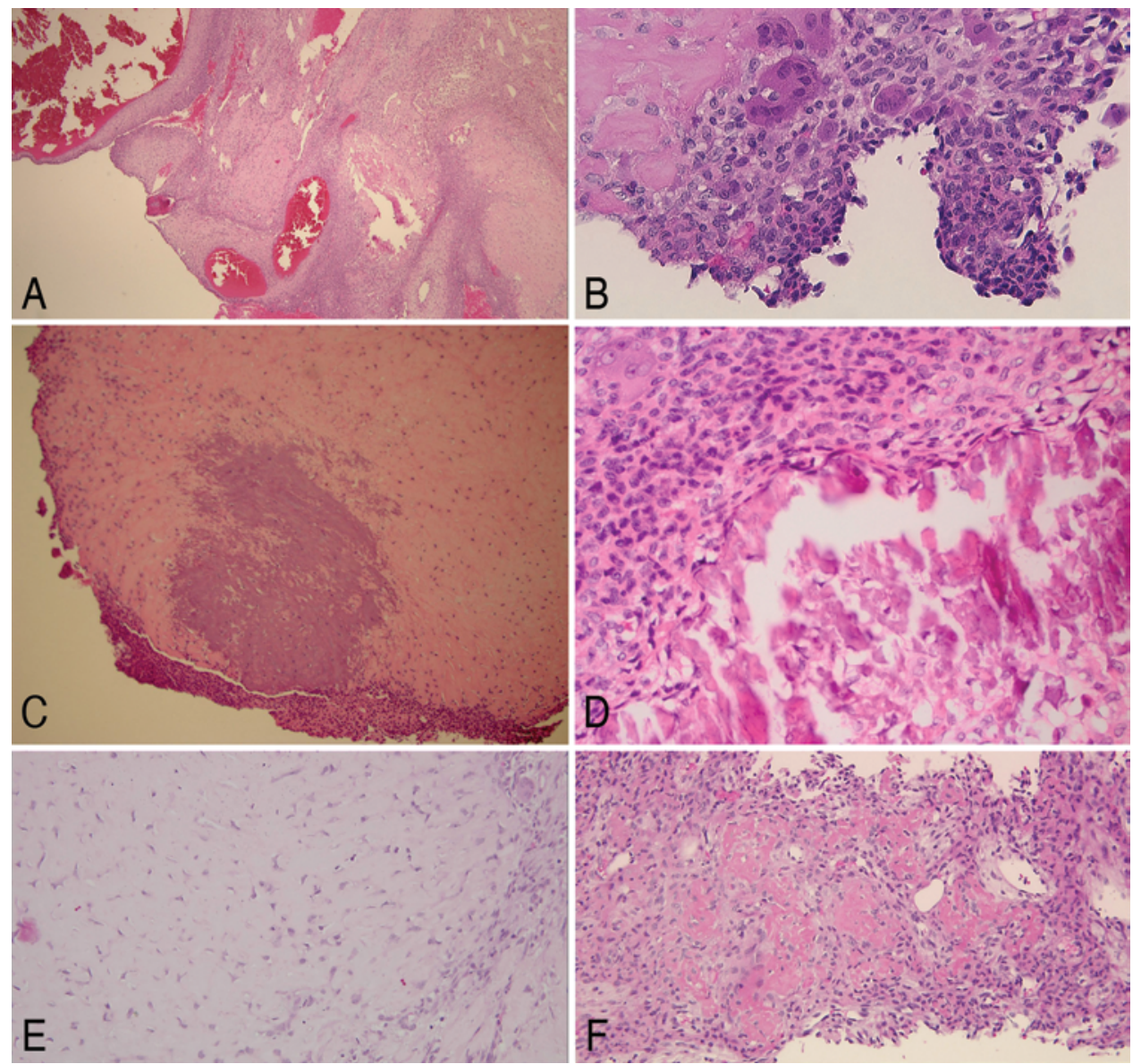

FIG. 2. Microscopic images of the tumor. Phosphaturic mesenchymal tumor with prominent vascularity and cellular areas with bland spindle- to stellate-shaped cells and scattered osteoclast-like giant cells (A and B). The stroma is eosinophilic and hyalinized with characteristic "grungy" calcification ( $C$ and $D)$, and focal chondroid and osteoid features ( $E$ and $F)$. H \& $E$, original magnification $\times 40(A) ; \times 400(B, C, D$, and F); $\times 200(E)$. Figure is available in color online only.

for that cohort). ${ }^{9}$ Ninety percent of PMT-MCTs present with hypophosphatemic osteomalacia due to excessive FGF23 expression, but in the absence of osteomalacia, the authors were able to make the diagnosis on the basis of histologically identical features plus FGF23 expression., ${ }^{9,16}$ Our differential diagnosis included chondromyxoid fibroma with $\mathrm{ABC}$. Further quantitative analysis (positive FGF23 on RT-PCR and negative USP6 on FISH) refined our diagnosis and confirmed PMT-MCT.

The great majority of PMT-MCTs are benign and have good prognosis with surgical excision; a wide surgical excision with clean margins is considered definitive treatment. ${ }^{14}$ However, rare cases of malignant and metastatic tumors have been reported, and because of potential death, caution should be exercised. Early on it was estimated that $90 \%$ of PMT-MCTs were benign; ${ }^{29}$ however, a later review of 32 cases found that $9.4 \%$ of benign tumors become malignant. ${ }^{9}$ This was based on cytology and 1 case of hematogenous metastasis to the lung. The report did not describe mortality. Morimoto et al. ${ }^{19}$ presented 2 cases of malignant pelvic PMTs presenting with oncogenic osteomalacia. In the first case, the patient harbored synchronous double cancer associated with thyroid cancer. The patient died after the tumor rapidly metastasized to the lung. The second patient experienced recurrence 2 years after open biopsy and complete resection and also died of metastatic tumor to the lung. ${ }^{19}$ Ogose et al. ${ }^{20}$ documented local recurrence and malignant transformation from benign PMT over a 17-year follow-up period. Pallavi et al..$^{21}$ reported a case of a 46-year-old man with PMT-MCT without evidence of oncogenic osteomalacia or phosphaturia, but the patient was found to have multiple osseous metastases throughout his body on initial workup. The authors noted that in the absence of standard treatment guidelines for metastatic PMTs, these tumors may be treated like metastatic softtissue sarcomas. Increased risk of recurrence or progression has been hypothesized to be associated with subtotal excision $^{17}$ or lengthy time from onset of symptoms to diagnosis. ${ }^{14}$ Tumor recurrence can be monitored with serial imaging using MRI, PET CT, indium-111 pentetreotide or octreotide scintigraphy, ${ }^{14}$ and laboratory workup including 24-hour urine phosphorus, serum phosphorus, serum calcium, serum $1-\mathrm{OH}$ and $1,25-\mathrm{OH}$ cholecalciferol, alkaline phosphatase, and serum FGF23, ${ }^{6}$ the last of which is regarded as the most direct serological marker for tumor activity. ${ }^{14}$ Our patient's symptoms resolved after excision, and she remained free of tumor recurrence on follow-up MRI.

A discussion of nonphosphaturic variants must be con- 
ducted in the context of their phosphaturic counterparts. PMTs are rare and difficult to diagnose; diagnosis is most often made after the onset of symptoms of osteomalacia. Indeed, PMTs were discovered because of their associated paraneoplastic effects. The typical patient will present with symptoms in the 3rd to 7th decades of life, and definitive diagnosis is reached at an average of 7.2 years (range 2-12 years) after onset of symptoms..$^{14}$ Upon suspicion, workup for patients presenting with typical TIO symptoms should include first characterizing the laboratory profile (phosphate, calcium, alkaline phosphate, vitamin $\mathrm{D}$, and serum FGF23). Localizing the tumor is the next task and relies on imaging modalities such as radiography, MRI, bone scanning, and octreotide scanning. If possible, surgical excision allows for further histological and immunohistochemical analyses, and serial monitoring with laboratory evaluations and imaging can document a resolution of symptoms as well as alert to the rare chance of recurrence.

While TIO is not specific to PMT, since it is associated with 140 different types of tumors, ${ }^{14}$ most TIOs are of a single histopathological entity. ${ }^{9}$ Progress has been made in detecting FGF23 and distinguishing TIO from PMTs versus from other etiologies..$^{15}$ Imel et al. ${ }^{13}$ documented the sensitivity of FGF23 in TIO using 3 different enzymelinked immunosorbent assay (ELISA) methods. In all 13 patients with confirmed tumors, sensitivity was $100 \%$ using the Kainos Intact assay. Of all 22 patients combined (those with suspected TIO and those with confirmed tumors), 19 of 22 (89\%) exhibited elevated FGF23 concentrations. ${ }^{13}$ In a cross-sectional study, Endo et al. ${ }^{7}$ showed clinical utility of FGF23 measurements by demonstrating an ability to differentiate TIO and XLH (X-linked hypophosphatemic rickets) from hypophosphaturic diseases of other etiology (vitamin D deficiency, Fanconi's syndrome, and Cushing's syndrome). In patients with other etiologies, FGF23 was undetectable in most patients, regardless of medical treatment. Coupling these findings with low serum phosphate using age-dependent reference ranges, these authors proposed diagnostic criteria to distinguish TIO and XLH from other hypophosphatemic diseases. ${ }^{7}$ As noted above, Folpe et al. reported that the role of RT-PCR identification of FGF23 was significant ${ }^{9}$ and facilitated the diagnosis of PMT-MCT when combined with histological features in $90 \%$ of cases. ${ }^{9,16}$ Bahrami et al. ${ }^{2}$ examined RT-PCR analysis for FGF23 and found that 94\% of PMTs with TIO and $75 \%$ of histologically diagnosed tumors without TIO were positive. They postulated that the absence of TIO in this nonphosphaturic group might be explained by presymptomatic detection, low levels or nonfunctional form of FGF23 expression, unknown compensatory mechanisms, absent presecretion phosphate levels, or lack of clinical recognition. ${ }^{2,9}$ In this same study, 3 of 23 non-PMT controls were positive, specifically in 2 chondromyxoid fibromas (CMFs) and $1 \mathrm{ABC}$, all of which were typical histologically and radiographically of their respective tumor type. Another study showed positive FGF23 expression in 29\% of 7 CMFs and in $44 \%$ of 16 ABCs..$^{11}$ Most recently, Carter et al. ${ }^{5}$ published on a potentially valuable diagnostic adjuvant of chromogenic in situ hybridization (CISH) assay for FGF23 mRNA in
PMT and found a sensitivity of $96 \%$ and a specificity of $100 \%$. Their study looked at 25 patients with classic PMTs with clinically documented TIO and compared CISH results with 40 control cases of other types of tumors. Unfortunately, this study did not examine any cases exhibiting morphological features of PMT but without known TIO ("nonphosphaturic variants of PMT"). ${ }^{2}$ This subtype can be difficult to quantify without pre- and postoperative laboratory results ${ }^{12,18}$ but can convey adverse effects. ${ }^{26}$ Ultimately, nonphosphaturic variants of PMT as well as PMT with TIO are able to be diagnosed by histological morphological findings even without ancillary FGF23 testing. ${ }^{11}$ FGF23 mRNA expression by RT-PCR or CISH further supports the diagnosis of PMT.

As our patient was much younger than typical presentation and without the paraneoplastic syndrome, it is thought that her tumor, though positive for FGF23 production, did not have time to develop the characteristic TIO symptoms. ${ }^{2}$ The rapid onset of the patient's other symptoms related to her tumor location led to its fortuitous discovery and allowed for prompt excision; final characterization of the tumor was accomplished later. The histological findings coupled with the confirmation of FGF23 expression in the brain by RT-PCR established our diagnosis with a high probability.

As noted by Bower and colleagues, though extremely rare to present without tumor-induced osteomalacia or phosphaturia, PMT-MCT may present intracranially without such typical associated symptoms. ${ }^{3}$ Of the only 8 reported intracranial cases, $7(88 \%)$ occurred in the anterior fossa. ${ }^{1,6,10,17,23,24,28}$ Of these 8 tumors, only 6 were ultimately diagnosed as PMT: ${ }^{17}$ regarding the 2 remaining tumors, one was ultimately diagnosed as hemangiopericytoma ${ }^{24}$ and the other was not resected. ${ }^{1}$ Only 3 intracranial hemorrhagic cases have been reported; ${ }^{10,17,28}$ an elevated index of suspicion for potential recurrence should be noted with cases that initially present as hemorrhagic. ${ }^{17}$ As noted in the literature, PMT-MCT is diagnosed much more frequently in adult patients, although it has been found in pediatric patients; the youngest patient diagnosed with an intracranial PMT-MCT was 28 years of age. The mean patient age at diagnosis calculated from these 3 studies was 50.75 years..$^{10,17,28}$

\section{Conclusions}

PMT-MCTs are benign tumors that can involve the brain. They may occur in the pediatric population. In our case, resection was safe and effective.

\section{References}

1. Andreopoulou P, Dumitrescu CE, Kelly MH, Brillante BA, Cutler Peck CM, Wodajo FM, et al: Selective venous catheterization for the localization of phosphaturic mesenchymal tumors. J Bone Miner Res 26:1295-1302, 2011

2. Bahrami A, Weiss SW, Montgomery E, Horvai AE, Jin L, Inwards CY, et al: RT-PCR analysis for FGF23 using paraffin sections in the diagnosis of phosphaturic mesenchymal tumors with and without known tumor induced osteomalacia. Am J Surg Pathol 33:1348-1354, 2009

3. Bower RS, Daugherty WP, Giannini C, Parney IF: Intracranial phosphaturic mesenchymal tumor, mixed connective 
tissue variant presenting without oncogenic osteomalacia. Surg Neurol Int 3:151, 2012

4. Carpenter TO: Oncogenic osteomalacia-a complex dance of factors. N Engl J Med 348:1705-1708, 2003

5. Carter JM, Caron BL, Dogan A, Folpe AL: A novel chromogenic in situ hybridization assay for FGF23 mRNA in phosphaturic mesenchymal tumors. Am J Surg Pathol 39:75-83, 2015

6. David K, Revesz T, Kratimenos G, Krausz T, Crockard HA: Oncogenic osteomalacia associated with a meningeal phosphaturic mesenchymal tumor. Case report. J Neurosurg 84:288-292, 1996

7. Endo I, Fukumoto S, Ozono K, Namba N, Tanaka H, Inoue $\mathrm{D}$, et al: Clinical usefulness of measurement of fibroblast growth factor 23 (FGF23) in hypophosphatemic patients: proposal of diagnostic criteria using FGF23 measurement. Bone 42:1235-1239, 2008

8. Evans DJ, Azzopardi JG: Distinctive tumours of bone and soft tissue causing acquired vitamin-D-resistant osteomalacia. Lancet 1:353-354, 1972

9. Folpe AL, Fanburg-Smith JC, Billings SD, Bisceglia M, Bertoni F, Cho JY, et al: Most osteomalacia-associated mesenchymal tumors are a single histopathologic entity: an analysis of 32 cases and a comprehensive review of the literature. Am J Surg Pathol 28:1-30, 2004

10. Gonzalez-Compta X, Mañós-Pujol M, Foglia-Fernandez M, Peral E, Condom E, Claveguera T, et al: Oncogenic osteomalacia: case report and review of head and neck associated tumours. J Laryngol Otol 112:389-392, 1998

11. Graham R, Krishnamurthy S, Oliveira A, Inwards C, Folpe AL: Frequent expression of fibroblast growth factor-23 (FGF23) mRNA in aneurysmal bone cysts and chondromyxoid fibromas. J Clin Pathol 65:907-909, 2012

12. Honda R, Kawabata Y, Ito S, Kikuchi F: Phosphaturic mesenchymal tumor, mixed connective tissue type, non-phosphaturic variant: report of a case and review of 32 cases from the Japanese published work. J Dermatol 41:845-849, 2014

13. Imel EA, Peacock M, Pitukcheewanont P, Heller HJ, Ward LM, Shulman D, et al: Sensitivity of fibroblast growth factor 23 measurements in tumor-induced osteomalacia. J Clin Endocrinol Metab 91:2055-2061, 2006

14. Ledford CK, Zelenski NA, Cardona DM, Brigman BE, Eward WC: The phosphaturic mesenchymal tumor: why is definitive diagnosis and curative surgery often delayed? Clin Orthop Relat Res 471:3618-3625, 2013

15. Lee JY, Imel EA: The changing face of hypophosphatemic disorders in the FGF-23 era. Pediatr Endocrinol Rev 10 (Suppl 2):367-379, 2013

16. Luo L, Low N, Vandervord J: Mandibular phosphaturic mesenchymal tumor-mixed connective tissue variant in a young girl. Cleft Palate Craniofac J 50:751-753, 2013

17. Mathis DA, Stehel EJ Jr, Beshay JE, Mickey BE, Folpe AL, Raisanen J: Intracranial phosphaturic mesenchymal tumors: report of 2 cases. J Neurosurg 118:903-907, 2013

18. Mavrogenis AF, Sakellariou VI, Soultanis K, Mahera H, Korres DS, Papagelopoulos PJ: A nonphosphaturic mesenchymal tumor mixed connective tissue variant of the sacrum. Orthopedics 33:851, 2010

19. Morimoto T, Takenaka S, Hashimoto N, Araki N, Myoui A, Yoshikawa H: Malignant phosphaturic mesenchymal tumor of the pelvis: A report of two cases. Oncol Lett 8:67-71, 2014

20. Ogose A, Hotta T, Emura I, Hatano H, Inoue Y, Umezu H, et al: Recurrent malignant variant of phosphaturic mesenchy- mal tumor with oncogenic osteomalacia. Skeletal Radiol 30:99-103, 2001

21. Pallavi R, Ravella PM, Gupta P, Popescu A: A case of phosphaturic mesenchymal tumor. Am J Ther 22:e57-e61, 2015

22. Prader A, Illig R, Uehlinger E, Stalder G: [Rickets following bone tumor.] Helv Paediatr Acta 14:554-565, 1959 (Ger)

23. Reis-Filho JS, Paiva ME, Lopes JM: August 2003: 47-yearold female with a 7-year history of osteomalacia and hypophosphatemia. Brain Pathol 14:111-112,115, 2004

24. Sandhu FA, Martuza RL: Craniofacial hemangiopericytoma associated with oncogenic osteomalacia: case report. J Neurooncol 46:241-247, 2000

25. Shimada T, Mizutani S, Muto T, Yoneya T, Hino R, Takeda $\mathrm{S}$, et al: Cloning and characterization of FGF23 as a causative factor of tumor-induced osteomalacia. Proc Natl Acad Sci U S A 98: 6500-6505, 2001

26. Uchihashi K, Nishijima-Matsunobu A, Matsuyama A, Yamasaki F, Tanabe T, Uemura T, et al: Phosphaturic mesenchymal tumor, nonphosphaturic variant, causing fatal pulmonary metastasis. Hum Pathol 44:2614-2618, 2013

27. Ungari C, Rocchi G, Rinna C, Agrillo A, Lattanzi A, Pagnoni M: Hypophosphaturic mesenchymal tumor of the ethmoid associated with oncogenic osteomalacia. J Craniofac Surg 15:523-527, 2004

28. Uno T, Kawai K, Kunii N, Fukumoto S, Shibahara J, Motoi T, et al: Osteomalacia caused by skull base tumors: report of 2 cases. Neurosurgery 69:E239-E244, 2011

29. Weidner N, Santa Cruz D: Phosphaturic mesenchymal tumors. A polymorphous group causing osteomalacia or rickets. Cancer 59:1442-1454, 1987

30. Weiss D, Bar RS, Weidner N, Wener M, Lee F: Oncogenic osteomalacia: strange tumours in strange places. Postgrad Med J 61:349-355, 1985

31. William J, Laskin W, Nayar R, De Frias D: Diagnosis of phosphaturic mesenchymal tumor (mixed connective tissue type) by cytopathology. Diagn Cytopathol 40 (Suppl 2):E109-E113, 2012

\section{Disclosures}

The authors report no conflict of interest concerning the materials or methods used in this study or the findings specified in this paper.

\section{Author Contributions}

Conception and design: Feiz-Erfan, Ellis. Acquisition of data: Ellis. Analysis and interpretation of data: Feiz-Erfan, Ellis, Lal, Nair. Drafting the article: Ellis. Critically revising the article: all authors. Reviewed submitted version of manuscript: all authors. Study supervision: Feiz-Erfan.

\section{Supplemental Information}

\section{Previous Presentations}

Portions of this work were presented in poster form in 2013 at the Greater Phoenix Academic Excellence Day.

\section{Correspondence}

Iman Feiz-Erfan, Division of Neurosurgery, Maricopa Medical Center, 2601 E. Roosevelt St., Phoenix, AZ 85008-4956. email: iman_feiz-erfan@dmgaz.org. 